\title{
Neural dynamics of audiovisual synchrony and asynchrony perception in 6-month-old infants
}

\section{Franziska Kopp* and Claudia Dietrich}

Center for Lifespan Psychology, Max Planck Institute for Human Development, Berlin, Germany

Edited by:

Vincent Reid, University of Durham, UK

\section{Reviewed by:}

Lisa Scott, University of

Massachusetts, USA

Moritz M. Daum, University of Zurich,

Switzerland

\section{*Correspondence:}

Franziska Kopp, Center for Lifespan Psychology, Max Planck Institute for

Human Development, Lentzeallee 94, 14195 Berlin, Germany.

e-mail:kopp@mpib-berlin.mpg.de
Young infants are sensitive to multisensory temporal synchrony relations, but the neural dynamics of temporal interactions between vision and audition in infancy are not well understood. We investigated audiovisual synchrony and asynchrony perception in 6-month-old infants using event-related brain potentials (ERP). In a prior behavioral experiment $(n=45)$, infants were habituated to an audiovisual synchronous stimulus and tested for recovery of interest by presenting an asynchronous test stimulus in which the visual stream was delayed with respect to the auditory stream by 400 ms. Infants who behaviorally discriminated the change in temporal alignment were included in further analyses. In the EEG experiment (final sample: $n=15$ ), synchronous and asynchronous stimuli (visual delay of $400 \mathrm{~ms}$ ) were presented in random order. Results show latency shifts in the auditory ERP components N1 and P2 as well as the infant ERP component Nc. Latencies in the asynchronous condition were significantly longer than in the synchronous condition. After video onset but preceding the auditory onset, amplitude modulations propagating from posterior to anterior sites and related to the Pb component of infants' ERP were observed. Results suggest temporal interactions between the two modalities. Specifically, they point to the significance of anticipatory visual motion for auditory processing, and indicate young infants' predictive capacities for audiovisual temporal synchrony relations.

Keywords: multisensory perception, audiovisual, infancy, ERP, synchrony, asynchrony

\section{INTRODUCTION}

In natural environments, continuous streams of multisensory stimuli are processed in the human brain. When these stimuli occur at the same spatial location and in temporal synchrony, perception as unitary events originating from the same source is very likely (Welch and Warren, 1980). Temporal synchrony seems to be a particularly strong binding cue (e.g., Spence and Squire, 2003). For audition and vision, neural activity in largescale networks has been found to interact with synchrony relations (e.g., Bushara et al., 2001; Macaluso et al., 2004; Miller and D'Esposito, 2005). Empirical evidence suggests that, within temporal proximity, stimuli from the two sensory modalities are pulled into temporal alignment and perceived as unitary events (e.g., Lewkowicz, 1996; Fendrich and Corballis, 2001). The dynamics by which the cognitive system creates synchronous versus asynchronous percepts have a certain degree of flexibility, depending on a variety of factors such as stimulus complexity, familiarity and experience, and repeated asynchrony presentation (e.g., Dixon and Spitz, 1980; Fujisaki et al., 2004; Vatakis et al., 2007; Petrini et al., 2009a). Importantly, these temporal binding mechanisms appear to undergo changes across the lifespan (Lewkowicz, 1996, 2010; Hay-McCutcheon et al., 2009).

Research in animals and humans suggests that multisensory interactions develop gradually during postnatal life, and that early experience is critical for the maturation of multisensory capabilities (e.g., Wallace et al., 2004; Putzar et al., 2007). Human infants are sensitive to temporal synchrony relations of auditory and visual events from early on. For example, Dodd (1979) demonstrated that 10- to 16-week-old infants looked longer at an experimenter reciting nursery rhymes when the lip movements and the speech sounds were presented with an asynchrony of $400 \mathrm{~ms}$ as compared to synchrony. But this ability is not limited to speech stimuli. In a habituation paradigm, infants as young as 4 weeks detected a change in audiovisual synchrony relations in naturalistic objects striking against a surface (Bahrick, 2001). Similarly, in a visualpreference task, 4-month-old infants preferred synchronous over asynchronous presentations of moving stuffed animals with the corresponding impact sounds (Spelke, 1979). Moreover, a number of behavioral studies have demonstrated that, starting from birth (Lewkowicz et al., 2010), human infants rely on audiovisual synchrony for intersensory matching (e.g., Bahrick, 1983; Lewkowicz, 1986, 1992, 2000; Lewkowicz et al., 2008). However, little is known about how the infant neural system creates a synchronous or asynchronous percept. Lewkowicz investigated the temporal window in which auditory and visual stimuli are bound together to a unitary event and found that infants in the first year of life are less sensitive to audiovisual asynchrony than adults. For instance, infants tolerate temporal disparities of auditory signals preceding visual events by about $300 \mathrm{~ms}$ in bouncing, sounding objects (Lewkowicz, 1996) and by $500 \mathrm{~ms}$ or more in speech stimuli (Lewkowicz, 2010) and still perceive simultaneity. Apparently, the greater asynchrony tolerance does not change between 2 and 10 months.

Much of the empirical evidence for infant responses to multisensory stimuli stems from behavioral research paradigms, such as visual paired-preference tasks or habituation-test procedures. However, little is known about the neural mechanisms 
underlying synchronous and asynchronous multisensory perception in the infant brain. Insight into the emergence of such percepts could help to take an infant perspective and to understand multisensory processes in the less experienced perceptual system. As an adequate method, non-invasive EEG measures have been useful in exploring the dynamics of perceptual and cognitive processes early in development. Here we assessed eventrelated brain potentials (ERP) that allow tracking of neural signatures with high temporal resolution and reveal dynamics of the neural activity underlying audiovisual temporal binding processes.

In human adults, ERPs were found to be modulated by multisensory interactions. In particular, the auditory components N1 and P2 were sensitive to audiovisual presentations (for reviews, see Fort and Giard, 2004; Besle et al., 2009). N1 and P2 amplitude suppressions have been observed in bimodal as compared to unimodal events (e.g., Besle et al., 2004; van Wassenhove et al., 2005; Stekelenburg and Vroomen, 2007). N1 and P2 latencies were shortened with audiovisual presentations, indicating faster processing in congruent multisensory events (van Wassenhove et al., 2005; Stekelenburg and Vroomen, 2007; Vroomen and Stekelenburg, 2009). ERP responses were also found to vary with temporal synchrony relations. Pilling (2009) replicated N1 and P2 amplitude decreases in synchronous audiovisual speech stimuli compared to unisensory auditory stimuli, but did not observe such amplitude suppressions when the auditory onset occurred $200 \mathrm{~ms}$ earlier. Similarly, Vroomen and Stekelenburg (2009) found that N1 and P2 amplitude modulations varied with the introduction of temporal asynchronies in audiovisual events. Importantly, some N1 and P2 modulations in multisensory events could only be observed when the stimuli contained salient visual inputs (van Wassenhove et al., 2005) or anticipatory visual motion, thus making the auditory onset predictable (Stekelenburg and Vroomen, 2007; Vroomen and Stekelenburg, 2009).

ERP research on multisensory perception early in ontogeny is still scarce. Hyde et al. (2010) investigated 3-month-olds' neural responses to bimodal versus unimodal presentation of circles and tones. In contrast to a number of findings in adults (see above), early auditory components were not sensitive to the experimental manipulation at this age. Rather, ERP responses differentiated between multisensory and unisensory presentations later in the analysis epoch (between 400 and $550 \mathrm{~ms}$ ). However, P2 mean amplitude modulations were observed in 5-month-olds (Hyde et al., 2011), suggesting increasing responsiveness of this auditory ERP component in the more mature perceptual system. In their study, Hyde et al. (2011) found greater P2 amplitudes for audiovisual synchronous stimuli including a speech sound and a static face as compared to asynchronous stimuli including the same speech sound with the static face onset delayed by $400 \mathrm{~ms}$. In a second experiment, larger P2 amplitudes were found for dynamic synchronous speech stimuli as compared to stimuli in which the facial movements in the visual stream did not correspond to the audio stream (Hyde et al., 2011). These findings demonstrate ERP modulations as a response to differences in multisensory perception in young infants. However, based on this research, conclusions about the processing of audiovisual temporal synchrony relations are difficult, because synchrony and asynchrony were confounded with static versus dynamic presentation, identity change, and manipulation of the visual onset.

In the present study, we aimed at exploring neural mechanisms of real audiovisual synchrony versus asynchrony perception in infancy by presenting dynamic video and sound stimuli. Thus, we avoided possible confounds with changes in static versus dynamic stimulus presentation (see Hyde et al., 2011). We applied a standardized infant-controlled habituation-test procedure to validate the individuals' capacities to detect a 400-ms asynchrony (see Lewkowicz, 1996) and assessed EEG responses to ecologically valid, audiovisual events (Stekelenburg and Vroomen, 2007) including socially relevant, communicative signals (De Gelder and Bertelson, 2003; Vatakis and Spence, 2006). We refrained from presenting speech stimuli to prevent interferences with individual language development processes in the preverbal infants. Instead, we produced videos of a person clapping her hands (Stekelenburg and Vroomen, 2007). Audiovisual asynchrony was achieved by delaying the content of the visual stream by $400 \mathrm{~ms}$ (see Materials and Methods and Figure 2) while keeping both the video and audio onset times and durations identical between the synchrony and asynchrony condition (cf. Doesburg et al., 2007). In contrast to several previous research paradigms (Pilling, 2009; Vroomen and Stekelenburg, 2009; Hyde et al., 2011), this setup avoided differences due to attentional shifts as orienting responses to stimulus onsets or offsets during the presentation, and attentional competition between the two sensory modalities (Talsma et al., 2010). Moreover, this design made it possible to keep the content of the stimulus constant, thereby avoiding a possible confound with change of congruency between the auditory and visual streams (e.g., Hyde et al., 2011). The magnitude of the audiovisual delay was piloted using a habituation paradigm. Earlier data have confirmed young infants' responses to a $400 \mathrm{~ms}$ audiovisual asynchrony in non-speech stimuli (Lewkowicz, 1996). The participants in the present study were 6-month-old infants. This age group was selected for several reasons. First, previous behavioral data have shown sensitivity to multisensory temporal relations in even younger infants (e.g., Bahrick, 2001; Lewkowicz et al., 2010). In other words, 6-month-olds are assumed to be able to detect the asynchrony. Second, it appears that the size of the temporal range of audiovisual integration does not change reliably in the first year of life (Lewkowicz, 1996). Third, as noted above, the general sensitivity of some early ERP components to the manipulation of multisensory phenomena seems to be more pronounced in infants older than 3 months (Hyde et al., 2010, 2011). Finally, 6-month-olds are awake and attentive enough to conduct an EEG experiment over a period of several minutes that yields sufficient data for ERP averaging. At the same time, the probability of spontaneous or even coordinated imitative behavior as a response to the hand-clapping stimuli is still very low at this age.

It is known that infant and adult ERP components may differ substantially in terms of amplitude, latency, or polarity (de Haan, 2007). Intra- and inter-individual variability is high in the immature ERP (de Haan, 2007), with variability in neural responses decreasing during the first months of life (Thomas et al., 1997). Despite these differences as compared to the adult ERP, the auditory components $\mathrm{N} 1$ and $\mathrm{P} 2$ have also been identified in infants. Wunderlich et al. (2006) reported (a) significantly longer N1 and 
P2 latencies in infants than in adults, (b) increases of N1 and P2 amplitude with age, and (c) more uniform scalp distributions in young infants and toddlers, but more focal distributions in older children and adults. For the present study, we expected similar results as those found in human adults during multisensory perception, that is, amplitude and/or latency modulations of the auditory components N1 and P2 (Stekelenburg and Vroomen, 2007; Pilling, 2009; Vroomen and Stekelenburg, 2009).

Regarding the multisensory nature of the stimuli, infantspecific ERP components related to visual recognition processes are relevant for this experiment. The most pronounced component is $\mathrm{Nc}$, a large negative deflection with a fronto-central distribution, peaking between 400 and $700 \mathrm{~ms}$ after stimulus onset. This component has been shown to relate to attentional processing, to orientation to salient stimuli, and to memory, as it was correlated with stimulus novelty (e.g., de Haan and Nelson, 1997; Ackles, 2008; Kopp and Lindenberger, 2011, 2012). A second, less well understood infant ERP component is $\mathrm{Pb}$, a positive deflection peaking between 250 and $450 \mathrm{~ms}$. It has been associated with processes of stimulus expectancy (e.g., Karrer and Monti, 1995) and with mechanisms related to the relevance of stimuli, particularly as coded over long-term retention periods (Kopp and Lindenberger, 2011, 2012). It is conceivable that neural mechanisms related to attention, as reflected in $\mathrm{Nc}$, and neural mechanisms related to expectancy, as reflected in $\mathrm{Pb}$, are both modulated by audiovisual synchrony versus asynchrony perception.

\section{MATERIALS AND METHODS PARTICIPANTS}

A total of 47 6-month-old infants were tested. All infants were born full-term ( $\geq 38$ th week), with birth weights of $2500 \mathrm{~g}$ or more. According to the respective caregiver's evaluation, all participants were free of neurological diseases, and had normal hearing and vision. In the behavioral habituation experiment, two infants were excluded due to an experimental error $(n=1)$ or failure to fulfill the fatigue criterion (see below; $n=1$ ). The final sample consisted of 45 infants ( 28 girls, 17 boys) with an age range of 170-195 days $(M=178.8$ days, $\mathrm{SD}=6.2$ days $)$.
Thirty infants who had been tested in the habituation experiment were not included in the final EEG analysis due to insufficient behavioral asynchrony discrimination in the habituation experiment (see below; $n=13$ ), or failure to reach the minimum requirements for adequate ERP averaging $(n=17)$, for example, because of excessive fussiness, movement artifacts, or insufficient visual fixation. The final sample of the EEG experiment included 15 infants ( 10 girls, 5 boys) with an age range between 171 and 193 days $(M=177.3$ days, $S D=5.5$ days $)$. The Ethics Committee of the Max Planck Institute for Human Development, Berlin approved this study. Informed written consent was obtained from the infants' caregivers.

\section{HABITUATION EXPERIMENT \\ Stimuli}

Videos were produced showing a female person who was highly trained in clapping her hands in a rhythm with equidistant intervals of $1000 \mathrm{~ms}$. She was instructed to synchronize her movements to audio signals presented to her left ear via a hidden headphone. A camera captured her face down to her shoulders, together with her hand movements in front of her face (see Figures 1 and 2), at a rate of 25 frames per second. Her facial expression was neutral with a slight smile to appear friendly to the child.

The parts of the footage that were most similar with respect to volume and precision of the clapping interval were chosen for further editing. Inter-clapping intervals deviated from $1000 \mathrm{~ms}$ by $\pm 21.7 \mathrm{~ms}$ on average. A synchronous and an asynchronous stimulus were created, each with a duration of $30 \mathrm{~s}$. In the asynchronous hand-clapping sequence, the visual stream was delayed with respect to the sound by $400 \mathrm{~ms}$ (10 frames). A 30-s sequence of an age-appropriate child movie was presented at the beginning and at the end of the habituation experiment to control for signs of fatigue. Loudness of both the audiovisual clapping sequences and the child movie sequences was $53 \mathrm{~dB}$ SPL (A).

\section{Procedure}

Infants were tested in a quiet, acoustically shielded room, in an area surrounded by white curtains to prevent visual distraction.

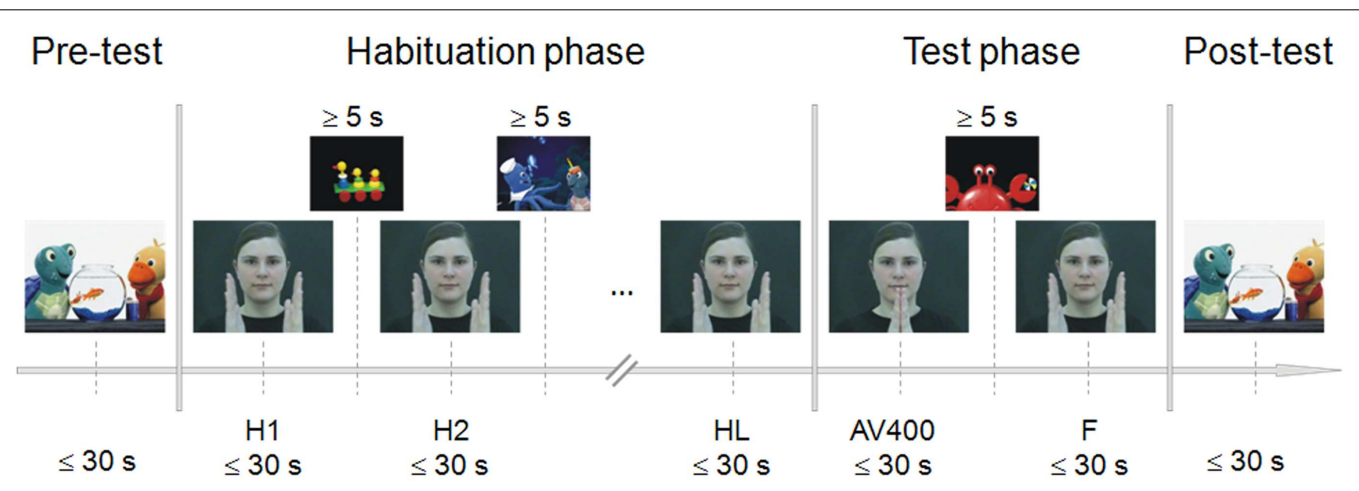

FIGURE 1 | Experimental procedure of the habituation paradigm. During pre- and post-test, a sequence of a child movie was presented to control for fatigue effects. Habituation was reached by repeated presentation of the audiovisual synchronous stimulus $(\mathrm{H} 1, \mathrm{H} 2, \ldots)$. After the last habituation trial $(\mathrm{HL})$, the novel asynchronous stimulus (AV400) was shown, followed again by the presentation of the familiar synchronous stimulus (F). Each experimental stimulus was presented as long as the infant looked at it or for a maximum duration of $30 \mathrm{~s}$. To attract the child's attention back to the screen, further sequences of the child movie were presented between habituation trials and between test trials. The child had to look at the screen for at least $5 \mathrm{~s}$ in order to continue with stimulus presentation. 


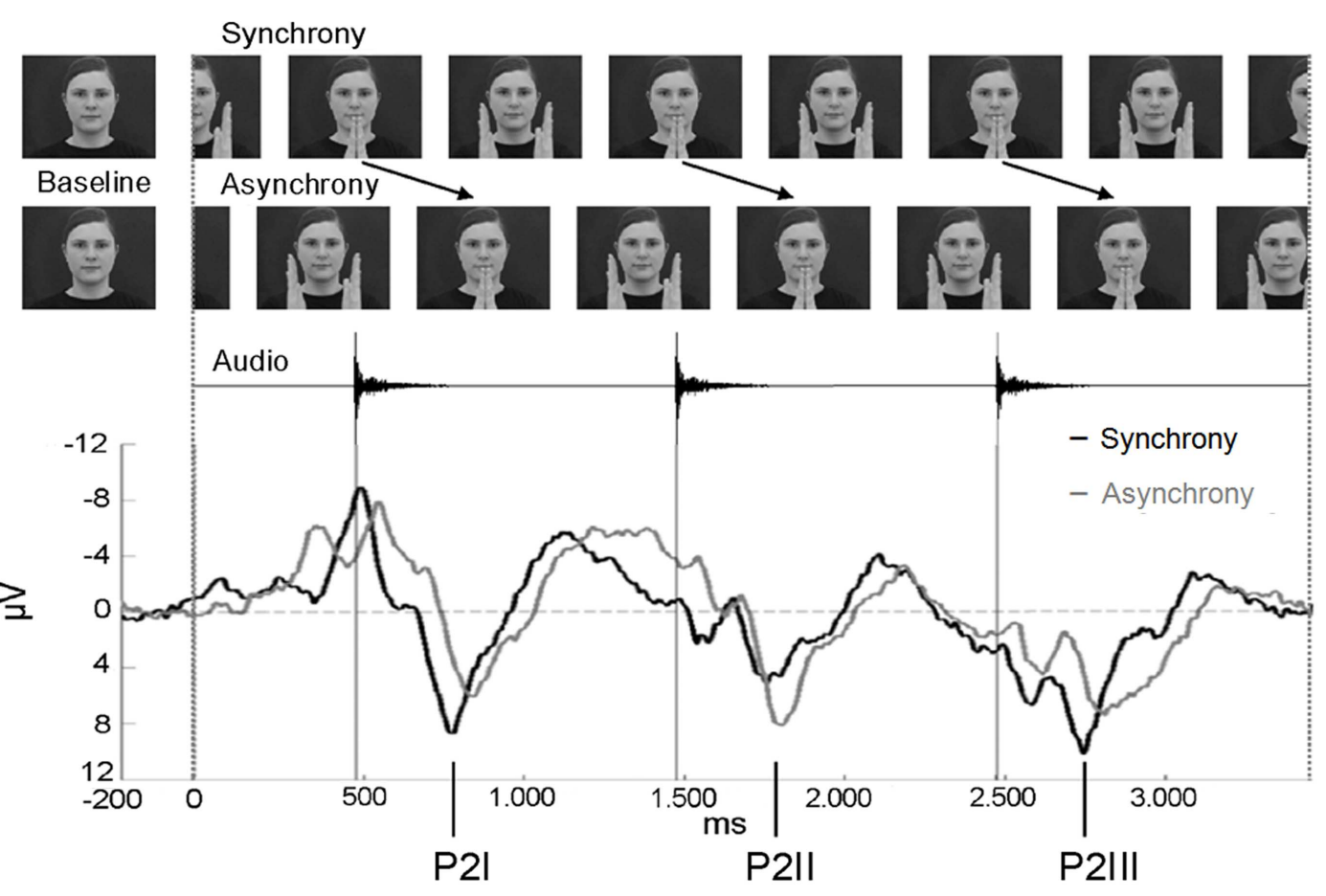

FIGURE 2 | Stimulus presentation of the EEG experiment and ERP epoch between $\mathbf{- 2 0 0}$ and $\mathbf{3 4 0 0} \mathbf{~ m s}$. In asynchronous trials, the visual stream was delayed by $400 \mathrm{~ms}$ to the auditory stream. The ERPs of the synchrony (black line) and the asynchrony condition (gray line) are averaged across anterior electrodes for the purpose of illustration $(n=15)$. Note that the auditory onset was at the same point in time in both conditions. The three P2 components elicited during the entire epoch are labeled P2I, P2II, and P2III.
The infant sat on the caregiver's lap. A computer screen was placed in front of the infant (distance $80 \mathrm{~cm}$ ), and two loudspeakers were placed to the left and to the right of the screen with a distance of approximately $100 \mathrm{~cm}$ to the infant's head. The infants' behavior was videorecorded to verify gaze durations during the experiment and again later off-line.

The procedure of the infant-controlled habituation paradigm was adapted from Lewkowicz $(1996,2010)$ and Flom and Bahrick (2007). The paradigm included a pre-test, habituation phase, test phase, and post-test (Figure 1). Pre- and post-tests served as controls for alertness before habituation trials and after test trials. For this purpose, sequences of the child movie were presented for as long as the child looked at them or for a maximum duration of $30 \mathrm{~s}$. Looking times of less than $5 \mathrm{~s}$ in the pre- and/or post-test led to the exclusion of the infant (fatigue criterion). The movie sequences were the same for pre- and post-test.

The habituation phase included repeated presentations of the synchronous clapping stimulus. Each of the habituation trials lasted as long as the child looked at the screen or for a maximum duration of $30 \mathrm{~s}$. An experimenter monitored the infant's gazing behavior. Whenever the infant looked away from the screen, she pressed a button and another sequence of the child movie appeared. The gaze away from the monitor had to last at least $1 \mathrm{~s}$ including a head movement. The child movie sequences between the habituation trials served to attract attention, and when the infant looked at them for at least $5 \mathrm{~s}$, the experimenter switched to the next habituation trial. The habituation criterion was reached when the mean gaze duration to the last three habituation trials was shorter than $50 \%$ of the mean gaze duration to the first three habituation trials. Thus, the minimum number of habituation trials was six.

In the test phase, the familiar synchronous stimulus (F) and the novel asynchronous test stimulus (AV400) were presented to test for recovery of interest. Again, the experimenter pressed a button when the child no longer looked at the screen or after a maximum of $30 \mathrm{~s}$. Sequences of the child movie appeared to attract the infant's attention, and when the child looked at the monitor for at least $5 \mathrm{~s}$, the experimenter switched again to the next test trial. This habituation-test procedure was applied to estimate individually whether or not the infant was able to discriminate the AV asynchrony of $400 \mathrm{~ms}$. Therefore, the order of test trial presentation was always the same across children. Infants saw and heard the last habituation trial (HL), then AV400, and then F before the post-test trial was presented.

\section{Data analysis}

An asynchrony discrimination score (ADS) based on individual gaze durations was calculated by dividing the looking time to $\mathrm{AV} 400$ by the mean value of looking times to $\mathrm{HL}$ and $\mathrm{F}^{1}$. Infants

\footnotetext{
${ }^{1}$ Due to high inter-individual variability in infant gazing behavior, significant response recovery can often be observed only in group-level data in which effects of different presentation orders are counterbalanced. Here, we chose an individual data approach including the same order of test trials for each infant. As this presentation
} 
with an ADS lower than 1.2 were not included in further EEG assessment. This criterion was chosen based on prior pilot data in our lab. It is rather strict considering that discrimination effects are usually revealed in group-level data. However, we aimed to establish that the individual had shown recovery of interest to the asynchronous test trial.

\section{EEG EXPERIMENT \\ Stimuli}

Two stimuli were created by cutting a sequence from the videos in which the female clapped her hands rhythmically at a rate of precisely $1000 \mathrm{~ms}$ (Figure 2). The synchronous stimulus consisted of four hand-clapping movements in synchrony with the corresponding hand-clapping sound. For the final analysis, EEG epochs comprised the first $3400 \mathrm{~ms}$ after stimulus onset with sounds occurring at 474,1474 , and $2474 \mathrm{~ms}$. The visual stimulus occupied a visual angle of $8.7^{\circ}$ when the female had extended both arms, and $7.1^{\circ}$ when her palms had clapped. The four hand-clapping sounds had an equalized sound pressure level of $48 \mathrm{~dB}$ SPL (A), each with a rise time of $10 \mathrm{~ms}$ and a fade-out period of $170 \mathrm{~ms}$.

The asynchronous stimulus was generated by including a visual delay of $400 \mathrm{~ms}$. That is, the video stream of the final EEG epochs showed the hands of the female clapping at 874,1874 , and $2874 \mathrm{~ms}$ after stimulus onset. A 400-ms video sequence of the continuous movement was added at the beginning and the video sequence of $400 \mathrm{~ms}$ at the end was cut out. In contrast, the time course of the auditory stream did not differ from the synchronous stimulus in terms of sound onset (at 474, 1474, and $2474 \mathrm{~ms}$ after video onset).

\section{Procedure}

The EEG experiment was conducted during the same test session. Right after the habituation paradigm, EEG equipment was set up. Surroundings, experimental setting, and video recording were the same as in the habituation experiment. Caregivers were briefed not to talk to the child or to point at the screen, nor to interact in any way with the child, and to avoid any movements to minimize EEG artifacts.

Synchronous and asynchronous stimuli were presented in random order in an event-related design. First, each trial started with an alternating sequence of an animated child movie to direct and maintain the child's attention to the screen. These animated movie sequences were randomly selected for each trial out of 20 sequences with varying durations between 3000 and $6000 \mathrm{~ms}$ (in steps of $500 \mathrm{~ms}$ ). Second, in both experimental conditions, a static photo of the female was presented for $1000 \mathrm{~ms}$ as a baseline. In order to make the transition between baseline and stimulus as smooth as possible, a snapshot of the subsequent clapping stimulus was adapted to create the baseline photo. The hands of the woman were removed and replaced by the background of the picture (Figure 2). For data analysis, the last $200 \mathrm{~ms}$ of the static image presentation were used as the pre-stimulus baseline for the ERP epochs. Third, the synchronous or the asynchronous clapping stimulus was presented for $4000 \mathrm{~ms}$. No more than three synchronous or three asynchronous trials were presented consecutively. Whenever the

mode could result in over- or underestimation of response recovery, we included gaze at the last habituation trial into the analysis. infant became fussy or did not look at the screen any longer, an age-appropriate animated movie was presented. When the infant attended to the screen again, the presentation of stimuli continued. The session ended when the infant's attention could no longer be attracted to the screen. Within the session, a maximum of 90 trials of synchronous and 90 trials of asynchronous stimuli were presented. Infants saw and heard on average 35.2 synchronous $(\mathrm{SD}=7.6)$ and 33.1 asynchronous stimuli $(\mathrm{SD}=5.5)$.

\section{EEG acquisition and analysis}

EEG signals were continuously recorded at 32 active electrodes with a sampling rate of $1000 \mathrm{~Hz}$ and amplified by a Brain Vision amplifier. The reference electrode was placed at the right mastoid, and the ground electrode at AFz. Signals at FP1 and FP2 were monitored to check for vertical eye movements, and signals at F9 and F10 were checked for horizontal eye movements. Impedances were kept below $20 \mathrm{k} \Omega$.

All trials in which the infant did not look at the screen were excluded from further analysis. EEG was re-referenced off-line to linked mastoids (Junghöfer et al., 1999). A bandpass filter was set off-line between 0.5 and $20 \mathrm{~Hz}$. ERP epochs comprised the 200-ms baseline before video onset followed by $3400 \mathrm{~ms}$ of video presentation. Artifacts due to eye or body movements or external sources were automatically discarded when voltage exceeded $\pm 120 \mu \mathrm{V}$. In addition, EEG signals were inspected visually to scan for and reject artifacts. A baseline correction to the 200-ms pre-stimulus baseline was performed. Finally, individual averages (ERP) and grand averages across subjects were calculated. For ERP analysis, infants contributed an average of 24.0 trials with synchronous ( $\mathrm{SD}=7.9$ ), and 23.0 trials with asynchronous stimuli $(\mathrm{SD}=7.4)$ to grand averages.

\section{RESULTS}

\section{BEHAVIORAL DATA}

Figure 3 shows mean gaze durations of the habituation-test paradigm. Infants reached habituation to the synchronous trial on average after 9.5 presentations. Average gaze durations to the animated child movie in the pre-test $(M=27115.8 \mathrm{~ms}, \mathrm{SD}=$ $5406.8 \mathrm{~ms})$ and post-test $(M=23821.3 \mathrm{~ms}, \mathrm{SD}=7503.8 \mathrm{~ms})$ were long, indicating high alertness during the whole test procedure.

The mean looking time to AV400 $(M=9272.8 \mathrm{~ms}, \mathrm{SD}=$ $5416.2 \mathrm{~ms})$ was reliably longer than to $\mathrm{F}(M=5646.7 \mathrm{~ms}$, $\mathrm{SD}=3416.1 \mathrm{~ms}), t(44)=4.26, p<0.001$. This result indicates that, on the group-level, infants showed recovery of interest after habituation to the synchronous stimulus and discriminated the AV asynchrony of $400 \mathrm{~ms}$. In line with the calculation of the ADS, an additional paired-samples $t$ test between gaze durations to AV400 and the mean of the gaze durations to HL and F $(M=5563.1 \mathrm{~ms}$, $\mathrm{SD}=2875.1 \mathrm{~ms}$ ) revealed a similar result, $t(44)=5.30, p<0.001$. That is, discrimination of the AV asynchrony of $400 \mathrm{~ms}$ could be established.

On average, $\mathrm{ADS}$ were $1.8(\mathrm{SD}=1.0, \mathrm{Min}=0.6, \mathrm{Max}=5.5)$ in this sample $(n=45)$. As explained above, infants with an ADS lower than 1.2 were excluded from the ERP analysis. Thirty-two infants $(71.1 \%)$ showed adequate $\mathrm{AV}$ asynchrony discrimination with a mean $\mathrm{ADS}$ of $2.2(\mathrm{SD}=1.0, \mathrm{Min}=1.2, \mathrm{Max}=5.5)$. A possible sampling bias with respect to sensitivity to audiovisual 
temporal synchrony relations cannot be fully excluded for the EEG experiment. However, non-significant visual recovery of interest to the AV400 trial after habituation to the synchronous stimulus cannot necessarily be referred to as a lack of discrimination of the asynchrony. Therefore, analyzing the neural activity patterns in infants who did not meet the ADS criterion seems preferable. Only $n=3$ out of those $n=13$ children provided EEG data sufficient for ERP analysis. Thus a meaningful statistical analysis was not possible.

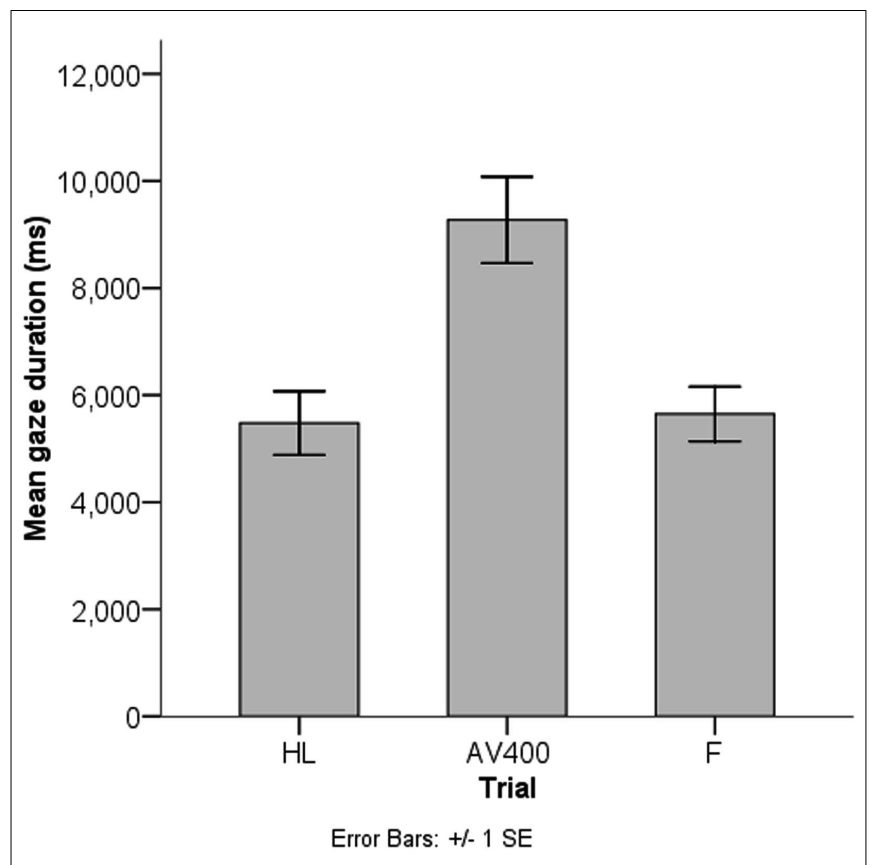

FIGURE 3 | Results of the behavioral habituation paradigm. Infants ( $n=45$ ) looked to the asynchronous test stimulus (AV400) for longer on average than to the familiar synchronous stimulus as shown for the last habituation trial $(\mathrm{HL})$ and the familiar test trial $(\mathrm{F})$.

\section{EEG DATA}

\section{ERP components and overview of analysis}

The most salient component was elicited as a response to the presentation of the auditory event (onsets at 474, 1474, and $2474 \mathrm{~ms}$, respectively) and may correspond to the adult $\mathrm{P} 2$. This component was analyzed for all three sounds within the $3400 \mathrm{~ms}$ epoch and termed P2I (interval: 700-900 ms after video onset), P2II (interval: $1700-1900 \mathrm{~ms}$ after video onset), and P2III (interval: 2700-2900 ms after video onset), respectively (Figure 2). P2 peaks occurred on average $284.7 \mathrm{~ms}$ after sound onset in synchronous stimuli and on average $346.0 \mathrm{~ms}$ in asynchronous stimuli. Prior to P2I, an auditory N1 was elicited (interval: $550-750 \mathrm{~ms}$ after video onset). The N1 activity partly overlapped with responses to the visual stimulation before the auditory onset (see Figure 4), however, the N1 peak could be identified and was therefore analyzed. N1 peaked on average at $156.3 \mathrm{~ms}$ after sound onset in synchronous stimuli and at $220.8 \mathrm{~ms}$ in asynchronous stimuli. In the time course of the epoch, ERP activity seemed to smear progressively (Figure 2). Therefore, only the pronounced P2 activities (P2I, P2II, P2III) were analyzed further. All other ERP components were evaluated within the interval of the first clapping sequence, that is, in the first $1000 \mathrm{~ms}$.

Other relevant ERP components were identified at the beginning of the EEG epochs, indicating significant aspects of audiovisual interaction at the beginning of the rhythmic stimulus. The negative component Nc (interval: $450-650 \mathrm{~ms}$ after video onset) was elicited primarily at anterior sites. ERP curves and topographic maps (Figures 4-6) suggest that activity differences between experimental conditions emerged between 200 and $400 \mathrm{~ms}$ at posterior electrodes with asynchronous stimuli eliciting more positive deflections. In the time interval of the visual $\mathrm{Pb}$ component between 300 and $400 \mathrm{~ms}$ after video onset, ERP differences were observed at anterior electrodes with a polarity reversal in asynchronous stimuli.

Separate repeated-measures analyses of variance (ANOVA) were performed for the following dependent variables: peak amplitudes and peak latencies of N1, P2I, P2II, P2III, and Nc and

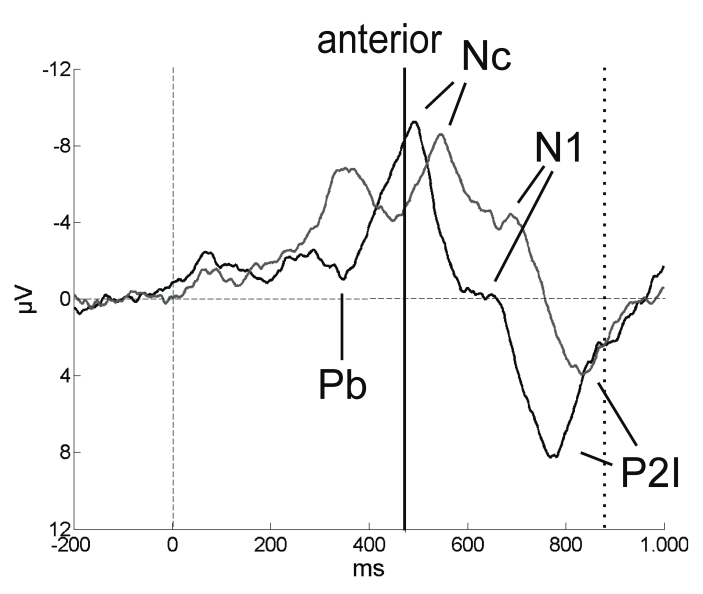

FIGURE 4 | ERP components in the first $1000 \mathrm{~ms}$ after video onset. The ERPs for synchrony (black line) and asynchrony (gray line) are averaged across all anterior and all posterior electrodes for the purpose of illustration $(n=15)$.

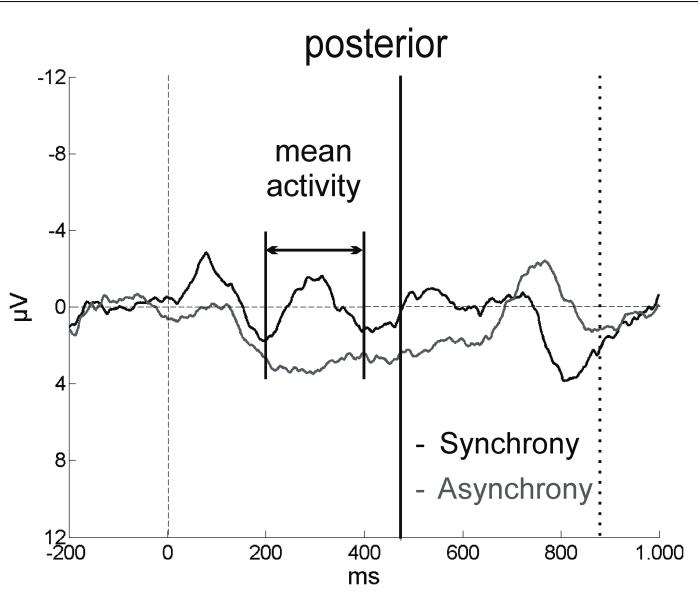

Solid vertical lines at $474 \mathrm{~ms}$ indicate the auditory onset in both experimental conditions. The visual clapping of the hands occurred at $874 \mathrm{~ms}$ in the asynchronous condition (indicated by dotted vertical lines). 

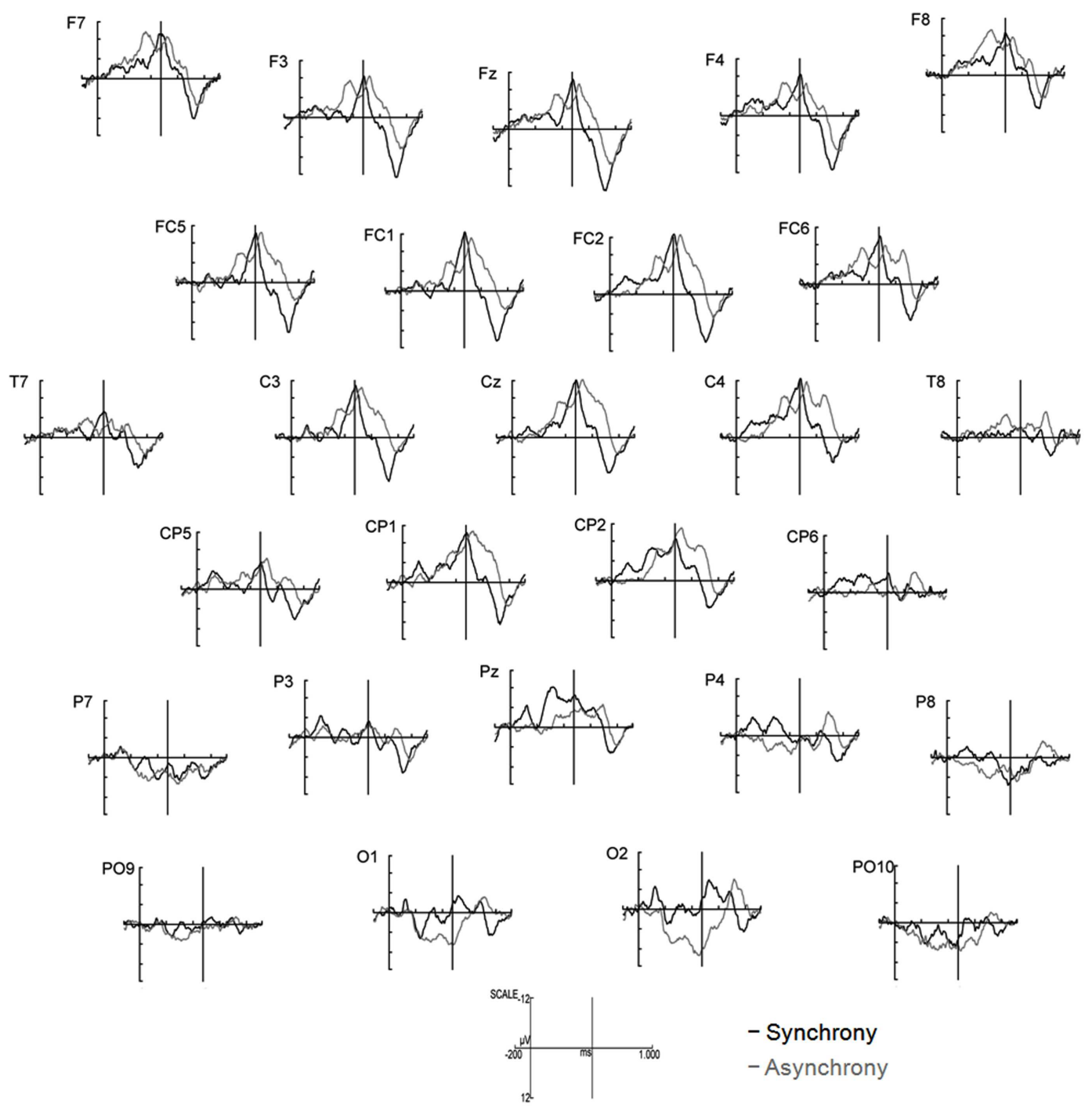

- Synchrony

- Asynchrony

FIGURE 5 | ERPs elicited by synchronous (black line) and asynchronous (gray lines) stimuli in the first 1000 ms of stimulus presentation ( $n=15)$. Note that the auditory event occurred at $474 \mathrm{~ms}$ in both experimental conditions (indicated by solid vertical lines).

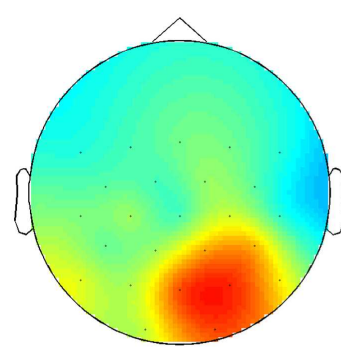

$200 \mathrm{~ms}-250 \mathrm{~ms}$

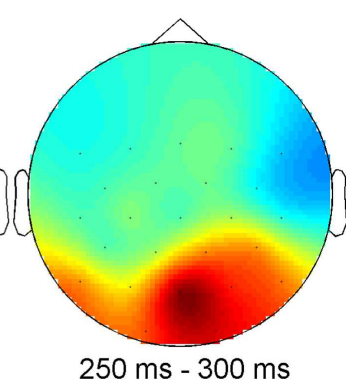

$250 \mathrm{~ms}-300 \mathrm{~ms}$

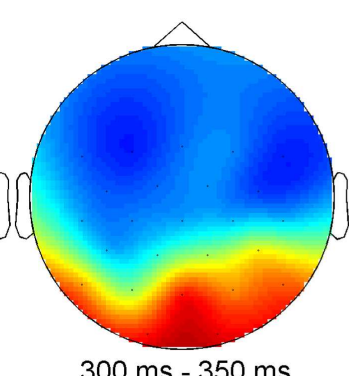

$300 \mathrm{~ms}-350 \mathrm{~ms}$

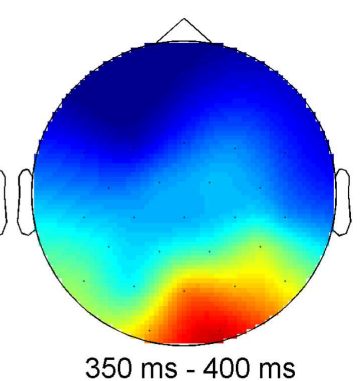

$350 \mathrm{~ms}-400 \mathrm{~ms}$

FIGURE 6 | Differential topographical map (asynchronous-synchronous trials) between 200 and 400 ms after stimulus onset ( $n=15$ ). Activity differences between the two stimuli emerged between 200 and $400 \mathrm{~ms}$ at posterior sites and between 300 and $400 \mathrm{~ms}$ at anterior electrodes. 
mean amplitudes of $\mathrm{Pb}$ and posterior activity (200-400 ms). For all analyses, the significance level of $\alpha=0.05$ was Bonferroniadjusted to control for multiple comparisons. Visual inspection suggested differential ERP activity between anterior and posterior sites (Figures $\mathbf{5}$ and 6). Therefore, topographic variability of the ERP components was tested first which resulted in the definition of regions of interest (ROI). This was done to prevent stimulus effects from remaining undetected in the less focal activity patterns of the infant ERP data. In addition, comparable ROIs related to similar experimental findings are still missing in the infancy literature. After the topographic analysis, specific effects of synchronous versus asynchronous stimuli were examined in the ROI electrodes while taking into account further possible lateralization effects.

\section{Topography}

Analyses of variances were performed including the withinsubjects factors Stimulus (synchronous versus asynchronous) and Region with the following regions defined by electrode lines from anterior to posterior: frontal $(\mathrm{F} 7, \mathrm{~F} 3, \mathrm{Fz}, \mathrm{F} 4, \mathrm{~F} 8)$, frontocentral (FC5, FC1, FC2, FC6), central/temporal (T7, C3, Cz, C4, T8), centro-parietal (CP5, CP1, CP2, CP6), parietal (P7, P3, Pz, $\mathrm{P} 4, \mathrm{P} 8)$, and parieto-occipital (PO9, O1, O2, PO10). Analyses revealed a main effect of Region on Nc amplitude, $F(5,70)=7.81$, $p<0.001, \eta^{2}=0.36$, mean posterior activity (200-400 ms), $F(5$, $70)=6.31, p<0.001, \eta^{2}=0.31$, and mean $\mathrm{Pb}$ activity, $F(5$, $70)=5.50, p<0.001, \eta^{2}=0.28$. More specifically, Nc amplitude was more negative in anterior than in posterior electrodes, whereas mean amplitude values between 200 and $400 \mathrm{~ms}$ and mean $\mathrm{Pb}$ amplitudes were on average negative at frontal, fronto-central, central/temporal, and centro-parietal electrodes but positive at parietal and parieto-occipital sites. The main effects of Region were qualified by reliable Region $\times$ Stimulus interactions on mean posterior activity $(200-400 \mathrm{~ms}), F(5,70)=4.92, p=0.001, \eta^{2}=0.26$, and mean $\mathrm{Pb}$ activity, $F(5,70)=5.68, p<0.001, \eta^{2}=0.29$. Accordingly, asynchronous stimuli elicited more negative voltages than synchronous stimuli in anterior electrodes but more positive amplitudes in parietal and parieto-occipital electrodes.

Furthermore, Region had a reliable main effect on latency of P2I, $F(5,70)=17.35, p<0.001, \eta^{2}=0.55$, P2II, $F(5,70)=25.59$, $p<0.001, \eta^{2}=0.65$, P2III, $F(5,70)=14.68, p<0.001, \eta^{2}=0.51$, and Nc, $F(5,70)=5.46, p<0.001, \eta^{2}=0.28$. In all four ERP components, latencies increased continuously from anterior to posterior. A reliable Region $\times$ Stimulus interaction was revealed for P2I latency, $F(5,70)=3.82, p=0.004, \eta^{2}=0.21$, and P2II latency, $F(5$, $70)=2.57, p=0.034, \eta^{2}=0.16$, with differential response latencies between synchronous and asynchronous stimuli at frontal, fronto-central, and central/temporal sites.

\section{ROI analyses}

Based on these statistical analyses and the topographic patterns (Figures 5 and 6), further analyses of specific Stimulus effects on $\mathrm{N} 1$, the $\mathrm{P} 2 \mathrm{~s}$, and $\mathrm{Nc}$ as well as mean $\mathrm{Pb}$ activity were performed at frontal, fronto-central and central/temporal electrodes, whereas analyses of mean posterior activity (200-400 ms) were performed at parietal and occipital electrodes (for descriptive statistics of the Stimulus effect, see Table 1). To assess possible variations in lateralization, the within-subject factor Hemisphere was included in the ANOVAs with the following ROI: left-hemisphere electrodes (anterior: F7, F3, FC5, T7, C3; posterior: P7, P3, PO9), midline electrodes (anterior: Fz, FC1, FC2, Cz; posterior: $\mathrm{Pz}, \mathrm{O} 1, \mathrm{O} 2$ ), and right-hemisphere electrodes (anterior: F4, F8, FC6, C4, T8; posterior: $\mathrm{P} 4, \mathrm{P} 8, \mathrm{PO} 10)$.

\section{N1}

Peak amplitude was more negative in asynchronous than in synchronous stimuli but this difference did not reach statistical significance. In contrast, $\mathrm{N} 1$ latency was reliably shorter in synchronous than in asynchronous trials, $F(1,14)=53.50, p<0.001, \eta^{2}=0.79$.

\section{P2I}

Analyses revealed that P2I amplitude was more positive in synchronous than in asynchronous trials but - similar to N1 amplitude - this difference did not reach statistical significance. P2I latency to synchronous stimuli was marginally shorter than to asynchronous stimuli, $F(1,14)=3.42, p=0.085, \eta^{2}=0.20$. However, follow-up analyses for Hemisphere indicated that the difference between experimental conditions was significant at anterior left- and marginal at right-hemisphere electrodes [left: $F(1$, $14)=5.38, p=0.036, \eta^{2}=0.28$; right: $F(1,14)=3.68, p=0.076$, $\left.\eta^{2}=0.21\right]$.

\section{P2II}

In contrast to P2I, peak amplitude was affected in P2II. Voltage was reliably higher in asynchronous than in synchronous trials in anterior midline electrodes, as indicated by a significant Hemisphere $\times$ Stimulus interaction, $F(2,28)=6.19, p=0.006$, $\eta^{2}=0.31$. Furthermore, Stimulus had a significant effect on P2II latency, $F(1,14)=9.21, p=0.009, \eta^{2}=0.40$, with shorter latencies in synchronous than in asynchronous stimuli.

\section{P2III}

No significant effects were found for P2III amplitude. Again, a significant main effect of Stimulus on P2III latency, $F(1$, 14) $=5.70, p=0.032, \eta^{2}=0.29$, indicated shorter latencies for synchronous than for asynchronous stimuli. Topographic variations in P2III latency were reflected in a reliable Hemisphere effect, $F(2,28)=3.67, p=0.038, \eta^{2}=0.21$. Accordingly, P2III activity peaks occurred earlier on average in anterior lefthemisphere $(M=2778.5 \mathrm{~ms})$ than in anterior midline electrodes $(M=2803.8 \mathrm{~ms})$.

\section{Nc}

Hemisphere had a significant main effect on Nc amplitude, $F(2$, 28) $=4.62, p=0.018, \eta^{2}=0.25$, with smaller (less negative) peak amplitudes in anterior left-hemisphere $(M=-10.2 \mu \mathrm{V})$ than anterior midline $(M=-13.8 \mu \mathrm{V})$ and anterior right-hemisphere $(M=-12.6 \mu \mathrm{V})$ electrodes. As with the P2s, Nc latency was reliably shifted between synchronous and asynchronous trials, $F(1$, 14) $=12.30, p=0.003, \eta^{2}=0.47$.

\section{Comparison of N1, P21, P2II, P2III, and Nc latency}

The significant peak latency differences between synchrony and asynchrony raise the question whether these temporal shifts vary 
Table 1 | Summary of descriptives of ERP components analyzed in synchronous and asynchronous trials in the specific regions of interest.

\begin{tabular}{|c|c|c|c|c|c|c|}
\hline & & & Mean & SD & Min & Max \\
\hline \multirow[t]{3}{*}{ N1 } & Amplitude $(\mu \mathrm{V})$ & Synchrony & -3.6 & 9.4 & -17.4 & 14.7 \\
\hline & & Asynchrony & -7.6 & 8.8 & -24.2 & 3.7 \\
\hline & & Asynchrony & $694.9 * *$ & 35.0 & 630.2 & 746.7 \\
\hline \multirow[t]{2}{*}{ P2I } & Amplitude $(\mu \mathrm{V})$ & Synchrony & 13.0 & 11.1 & -4.2 & 32.2 \\
\hline & & Asynchrony & $823.2^{+}$ & 110.4 & 643.0 & 1028.8 \\
\hline \multirow[t]{3}{*}{ P2II } & Amplitude $(\mu \mathrm{V})$ & Synchrony & 8.8 & 8.3 & -2.0 & 31.3 \\
\hline & & Asynchrony & $11.2^{++}$ & 10.7 & -2.0 & 44.0 \\
\hline & Latency (ms) & Synchrony & 1755.4 & 116.1 & 1579.8 & 2000.4 \\
\hline \multirow{2}{*}{ P2III } & Latency (ms) & Synchrony & 2766.4 & 113.0 & 2601.3 & 3006.9 \\
\hline & & Asynchrony & $2820.8^{*}$ & 94.5 & 2647.2 & 3039.5 \\
\hline \multirow[t]{4}{*}{ Nc } & Amplitude $(\mu \mathrm{V})$ & Synchrony & -12.3 & 8.2 & -26.6 & 6.5 \\
\hline & & Asynchrony & -12.2 & 9.2 & -25.1 & 8.4 \\
\hline & Latency (ms) & Synchrony & 526.8 & 69.9 & 449.8 & 664.8 \\
\hline & & Asynchrony & $601.4^{* *}$ & 71.2 & 500.5 & 709.1 \\
\hline \multirow[t]{2}{*}{$\mathrm{Pb}$} & Mean amplitude $(\mu \mathrm{V})$ & Synchrony & -1.3 & 9.0 & -19.5 & 12.7 \\
\hline & & Asynchrony & $-5.2^{*}$ & 7.9 & -18.6 & 14.6 \\
\hline Posterior activity (200-400 ms) & Mean amplitude $(\mu \mathrm{V})$ & Synchrony & 0.6 & 5.1 & -10.5 & 11.5 \\
\hline
\end{tabular}

Descriptives are collapsed across the factor Hemisphere; analysis of N1, P2I, P2II, P2III, Nc, and Pb in anterior (frontal, fronto-central, central/temporal) electrodes

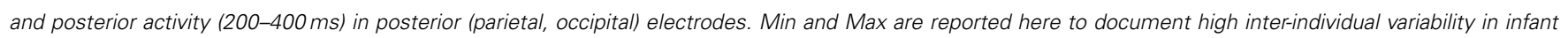
EEG data.

Asynchrony difference from Synchrony: ${ }^{*} p<0.05 .{ }^{*} p<0.01 .{ }^{+} p<0.05$ in left electrodes. ${ }^{++} p<0.01$ in midline electrodes.

within the trial. Figure 7 illustrates mean latency differences in Nc, N1, P2I, P2II, and P2III. A repeated-measures ANOVA including the within-subject factor Component (P2I versus P2II versus P2III) revealed no differences in the latency shifts between the individual P2 components, $F(2,28)=0.46, p=0.630$. Furthermore, including $\mathrm{N} 1$ and $\mathrm{Nc}$ in the same analysis yielded comparable results, $F(4,56)=0.50, p=0.737$. Thus, a constant difference in temporal alignment can be assumed. At the same time, these latency shifts between synchronous and asynchronous stimuli were significantly different from the AV delay of $400 \mathrm{~ms}$ in all five ERP components, as indicated by one-sample $t$ tests [N1: $t(14)=38.05, p<0.001$; P2I: $t(14)=15.24, p<0.001$; P2II: $t(14)=15.76, p<0.001$; P2III: $t(14)=15.40, p<0.001$; Nc: $t(14)=15.41, p<0.001]$.

\section{Mean Pb activity}

Asynchronous stimuli elicited reliably more negative mean amplitudes than did synchronous stimuli, $F(1,14)=5.85, p=0.030$, $\eta^{2}=0.30$ (Figures 5 and 6). The Hemisphere $\times$ Stimulus interaction was not significant, $F(2,28)=2.21, p=0.128$.

\section{Mean posterior activity (200-400 ms)}

Stimulus had a significant main effect on mean amplitude, $F(1$, $14)=6.13, p=0.027, \eta^{2}=0.30$, with more positive amplitudes in asynchronous than in synchronous trials. Visual inspection of posterior activity (200-400 ms) suggests more right-lateralized contributions to this effect (Figure 6). However, Hemisphere neither had a main effect nor interacted with Stimulus.

\section{Behavioral covariate}

The ADS, as an indicator for the magnitude of recovery of interest to AV400 after habituation to the synchronous stimulus, was entered into separate ANOVAs as a covariate. Dependent variables were the same as in the ROI analyses. There was a reliable Hemisphere $\times$ ADS interaction on mean posterior activity (200-400 ms), $F(2,26)=5.23, p=0.012, \eta^{2}=0.29$. That is, the higher the ADS, the more positive the mean activity between 200 and $400 \mathrm{~ms}$ was at posterior right electrodes [simple effect of $\mathrm{ADS}$ at posterior right electrodes: $F(1,13)=6.46$, $\left.p=0.025, \eta^{2}=0.33\right]$. This general lateralized activity pattern was independent of stimulus type. 


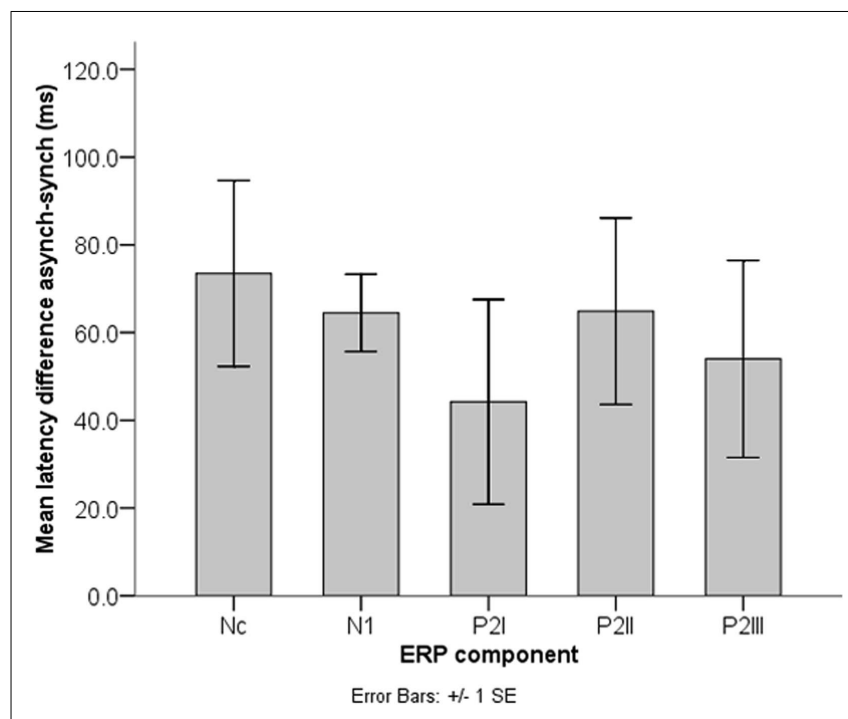

FIGURE 7 | Nc, N1, and P2 latency shifts ( $n=15)$. Latency differences between synchronous and asynchronous stimuli did not differ significantly between the ERP components and were always significantly smaller than the AV asynchrony of $400 \mathrm{~ms}$.

\section{DISCUSSION}

The results from the habituation-test paradigm of the present study confirm earlier work (Lewkowicz, 1996) by showing that, at the group level, infants in the first year of life are able to detect audiovisual asynchronies of $400 \mathrm{~ms}$ in non-speech stimuli. The degree of recovery of interest varied between infants. Interestingly, the magnitude of the behavioral ADS was correlated to neural activity as early as $200-400 \mathrm{~ms}$ at right posterior sites. In other words, the longer children gazed to the asynchrony, the larger a general neural responsiveness to this type of stimulus was, independent of temporal synchrony relations. With respect to the general infant population, however, one has to keep in mind that the result very likely overestimates the correlation as a result of the exclusion of children with fewer or no signs of dishabituation.

As predicted, ERP differences between synchronous and asynchronous events were evident in the auditory components $\mathrm{N} 1$ and $\mathrm{P} 2$. In adults, $\mathrm{N} 1$ and $\mathrm{P} 2$ amplitudes were shown to decrease in response to multisensory as compared to unisensory stimuli when both modalities were presented in synchrony (e.g., van Wassenhove et al., 2005; Stekelenburg and Vroomen, 2007; Besle et al., 2009) but not in asynchrony (Pilling, 2009). In the present study, $\mathrm{N} 1$ amplitudes tended to be less negative and P2II amplitudes were less positive in response to synchrony than to asynchrony. The amplitude reductions in synchronous audiovisual events resemble those described in adult research as reflecting neural interactions between the two modalities (Besle et al., 2009; Pilling, 2009). However, in the infant sample, N1 and P2 amplitude modulations were not statistically systematic (differences between synchronous and asynchronous stimuli were present in P2II but not significant in N1, P2I, and P2III; cf. Hyde et al., 2011). One could assume that this sort of pattern develops with increasing age. In addition, high intra- and inter-individual variability in infant ERPs or sample size may affect the results. Moreover, amplitude reductions may be of greater significance in bimodal interactions versus unimodal presentations than in the manipulation of temporal synchrony relations.

In contrast, the most striking and statistically robust result here is the constant temporal delay of auditory ERP peaks between synchronous and asynchronous stimuli (Figure 7), with on average significantly longer latencies in the asynchronous condition in $\mathrm{N} 1$ and the P2s. This finding is important given that the auditory events were presented at the same point in time in both conditions (sound onsets: 474, 1474, and $2474 \mathrm{~ms}$ ). Apparently, neural activity elicited by the auditory events was mostly delayed toward the corresponding visual activity. This temporal delay was maintained throughout the trial (Figure 7). As the behavioral data show, perceptual fusion is very unlikely (cf. Miller and D'Esposito, 2005; Stevenson et al., 2011). One could argue that these significant latency delays in the auditory ERP components may be an indicator for the emergence of an asynchronous percept on the behavioral level. Further, the temporal shift of auditory brain activity could reflect an attempt of the infant brain to operate against asynchrony (Hyde et al., 2011), despite the clear temporal disparity of vision and audition. At the same time, latency differences between synchronous and asynchronous stimuli in N1, P2I, P2II, and P2III were significantly smaller than the delay of $400 \mathrm{~ms}$ in the visual stream, thus suggesting a temporal interaction between the two modalities. Apparently 6 months or less of multisensory experience with synchronous events and/or exposure to the handclapping stimuli in the habituation task were sufficient to develop these processes.

A synchrony detection mechanism for binding across sensory modalities has been proposed in earlier research in adults (e.g., Navarra et al., 2005). Accordingly, temporally misaligned auditory and visual events are drawn into approximate temporal register, whereby the brain pulls auditory signals into temporal alignment with the corresponding visual stream (Vatakis et al., 2007; Navarra et al., 2009). The results of our experiment confirm these suggestions and show that similar mechanisms might operate in 6-month-old infants, at least in the present paradigm (but, see also Fort et al., 2002; Lewkowicz, 2010). Here, although the visual information lagged behind the corresponding auditory stream, infants also saw the hand movements of the asynchronous stimulus before sound onset, which might have initiated the delay in neural activity.

Similar latency effects in the adult ERP were reported to vary with predictability of auditory signals. That is, the temporal facilitation in N1 and P2 increased with higher salience of visual inputs (van Wassenhove et al., 2005). In our hand-clapping stimuli, predictability was high, as the sound onset could be predicted from the hand movements. Thus the latency shifts of the auditory N1 and $\mathrm{P} 2$ in the infant sample cannot be considered independent of prior visual motion. Indeed, results show that a significant latency shift was already revealed in the preceding Nc component. Again, longer latencies were observed in the asynchronous condition. Nc modulations have typically been associated with attentional mechanisms in infant visual recognition and memory (e.g., de Haan and Nelson, 1997; Ackles, 2008; Kopp and Lindenberger, 2011, 2012). Hence, the present Nc latency differences might imply an 
attentional shift in time between synchronous and asynchronous stimuli. The temporal course of the ERP pattern makes it very unlikely that Nc activity is a direct response to the physical sound onset. Rather the latency shift might be a result of the anticipated sound onset in each of the two conditions. There was on average no significant difference in the magnitude of the latency shifts between $\mathrm{Nc}, \mathrm{N} 1$, and the $\mathrm{P} 2 \mathrm{~s}$, which indicates that temporal activity relations between vision and audition were maintained throughout the rhythmic stimulus, once they had been established after video onset. The fact that these relations were present even before the auditory onset suggests prediction or anticipation processes in the infant brain that might trigger neural activity in the remaining stimulus epoch.

In fact, the data of this experiment show that differential activation between synchrony and asynchrony perception began as early as $200 \mathrm{~ms}$ after video onset (approximately $270 \mathrm{~ms}$ before sound onset). It propagated from posterior to anterior sites (Figure 6). Audiovisual asynchrony effectively elicited a polarity reversal of the $\mathrm{Pb}$ component in the anterior ERP. The functional significance of $\mathrm{Pb}$ in infant visual recognition paradigms is still under investigation. It has been related to mechanisms of stimulus expectancy, particularly to the probability of occurrence of an event (Karrer and Monti, 1995; Kopp and Lindenberger, 2011, 2012). Here, one could argue that the visual recognition process might be altered and that $\mathrm{Pb}$ modulations indicate differences in the infants' expectation of the hand-clapping movement. However, the visual stream in the asynchrony condition was not per se an odd or deviant stimulus. Instead, it was the same biologically possible motion as in the synchrony condition, and it was also presented with the same frequency during the experimental session. Hence, $\mathrm{Pb}$ activity differences between the two conditions suggest that the visual motion was perceived relative to the auditory event that occurred later in the trial and that could be expected at the same time point in both conditions. In other words, the 6-month-old infants of the present sample might have predicted audiovisual temporal synchrony relations of the current event (see also Petrini et al., 2009b). Future studies could include other visual control conditions to explore these dynamics further, provided that infants' attention allows for a longer test session. It is known from infant ERP research that, in visual recognition paradigms, unisensory stimuli with comparable onset time and duration elicit $\mathrm{Pb}$ activity with comparable polarity and latency and typically elicit amplitude modulations due to stimulus characteristics (e.g., Karrer and Monti, 1995; Hill Karrer et al., 1998; Webb et al., 2005). Hence one would assume that the mere temporal delay of the content of the visual stream should elicit similar $\mathrm{Pb}$ amplitude modulations in unisensory visual stimuli, but not a polarity reversal followed by Nc latency shifts. In other words, it is fair to conclude that the present ERP pattern of the asynchronous condition before sound onset $(474 \mathrm{~ms})$ is likely to reflect aspects of stimulus processing that are related to the multisensory nature of the event.

In contrast to earlier ERP findings on multisensory perception in 5-month-old infants (Hyde et al., 2011), the present data did not show consistent P2 amplitude modulations, and differences were also found in other ERP components. However, as noted in the introduction, the present paradigm investigating the perception of temporal synchrony relations was not directly comparable to the earlier work. With a static visual input in Hyde et al. (2011), Nc peak latency differences corresponding to the magnitude of the stimulus asynchrony were found. With a dynamic but modified visual input, no peak latency differences were observed. In contrast, using dynamic audiovisual presentations with temporally shifted visual content in our study, $\mathrm{Nc}$ and $\mathrm{P} 2$ latencies showed temporal interaction patterns, indicating dynamic perceptual binding mechanisms.

Next to the stimulus presentation, several other variables may potentially contribute to our results. First, type and complexity of the stimuli could be a source of variability. For example, it is known that perceptual binding in the temporal range of audiovisual integration is different for speech and non-speech events, both in adults (Dixon and Spitz, 1980) and in infants (Lewkowicz, 2010). Here, we used social non-speech stimuli. However, ERP modulations due to audiovisual interactions in adults were found to be similar between speech- and non-speech stimuli (Stekelenburg and Vroomen, 2007; Vroomen and Stekelenburg, 2009). Also, as noted above, anticipatory visual motion affected auditory N1 and P2 activity modulations in adults (Stekelenburg and Vroomen, 2007; Vroomen and Stekelenburg, 2009). Preceding visual movement reduced the uncertainty about an auditory onset (Besle et al., 2009), and with increased saliency of the visual input, predictability of an auditory signal also increased (van Wassenhove et al., 2005). It would certainly be interesting to investigate how the infant brain responds to stimuli without such anticipatory visual motion (cf. Stekelenburg and Vroomen, 2007). Furthermore, taking the infants' relatively short multisensory experience into account, the question arises how neural responses to temporal synchrony relations would look with completely novel stimuli. In other words, the present results are likely to reflect - at least to some extent - the influence of cumulative experience with the stimuli during the habituation and EEG paradigm. In addition to the present experimental manipulation, further control conditions could be helpful to elucidate the dynamics of multisensory processing. However, in infant samples, multiple conditions and repeated stimulus presentation may produce unacceptably high attrition rates and may have an effect on the ERP findings and their generalizability (for further discussion, see Snyder et al., 2002; Stets et al., 2012). Finally, in order to understand developmental changes of multisensory perception, future studies should investigate EEG activity in younger and older children and in adults. Taking general neural maturation processes into account, the present paradigm could help identify indicators of audiovisual temporal perception processes.

In the present study, visual and auditory ERP activity overlapped. Hence, when aiming for the separation of audition and vision and the application of an additive model (cf. Foxe and Molholm, 2009), a clear dissociation between the ERP components of each individual modality may be difficult (e.g., see discussion in Talsma et al., 2009). In addition, a comparison of ERP activity time-locked to the visual clapping would be complicated because of the lack of comparable stimulus onsets, ERP component activity relative to stimulus onset, and baseline activity (see Figure 4). The two experimental conditions in our study differed only in the temporal content of the visual input while visual and auditory onsets and sequence lengths did not vary. As 
the time course of the overlap of unisensory modalities is highly similar between synchronous and asynchronous stimuli, a direct comparison of ERP peaks between conditions seems valid. It is important to note that differences between the conditions already arose before the onset of auditory input. Hence, an overlap of visual and auditory ERP components alone cannot explain the neural activity patterns without taking into account temporal synchrony relations. Furthermore, from the perspective of ecological validity, one could argue that a purely unisensory input is rather unusual outside the laboratory. Here, the aim was to study multisensory stimulus processing as it could occur in everyday situations. For example, in a video internet call, the visual stream may be delayed with respect to the auditory signal due to technical constraints, and simultaneity perception might be difficult or impossible. We think that the brain adapts perfectly to naturalistic stimuli, and developmental mechanisms should be investigated in such contexts. This study shows that the perceptual system does not need years of multisensory experience

\section{REFERENCES}

Ackles, P. (2008). Stimulus novelty and cognitive-related ERP components of the infant brain. Percept. Mot. Skills 106, 3-20.

Bahrick, L. E. (1983). Infants' perception of substance and temporal synchrony in multimodal events. Infant Behav. Dev. 6, 429-451.

Bahrick, L. E. (2001). Increasing specificity in perceptual development: infants' detection of nested levels of multimodal stimulation. J. Exp. Child. Psychol. 79, 253-270.

Besle, J., Bertrand, O., and Giard, M.-H. (2009). Electrophysiological (EEG, sEEG, MEG) evidence for multiple audiovisual interactions in the human auditory cortex. Hear. Res. 258, 143-151.

Besle, J., Fort, A., Delpuech, C., and Giard, M.-H. (2004). Bimodal speech: early suppressive visual effects in human auditory cortex. Eur. J. Neurosci. 20, 2225-2234.

Bushara, K. O., Grafman, J., and Hallett, M. (2001). Neural correlates of auditory-visual stimulus onset asynchrony detection. J. Neurosci. 21, 300-304.

De Gelder, B., and Bertelson, P. (2003). Multisensory integration, perception and ecological validity. Trends Cogn. Sci. (Regul. Ed.) 7, 460-467.

de Haan, M. (2007). Infant EEG and Event-Related Potentials. Hove: Psychology Press.

de Haan, M., and Nelson, C. A. (1997). Recognition of the mother's face by six-month-old infants: a neurobehavioral study. Child Dev. 68, 187-210.

Dixon, N. F., and Spitz, L. (1980). The detection of auditory visual desynchrony. Perception 9, 719-721.
Dodd, B. (1979). Lip reading in infants: attention to speech presented in- and out-of-synchrony. Cogn. Psychol. 11, 478-484.

Doesburg, S. M., Emberson, L. L., Rahi, A., Cameron, D., and Ward, L. M. (2007). Asynchrony from synchrony: long-range gamma-band neural synchrony accompanies perception of audiovisual speech asynchrony. Exp. Brain Res. 185, 11-20.

Fendrich, R., and Corballis, P. M. (2001). The temporal cross-capture of audition and vision. Percept. Psychophys. 63, 719-725.

Flom, R., and Bahrick, L. E. (2007). The development of infant discrimination of affect in multimodal and unimodal stimulation: the role of intersensory redundancy. Dev. Psychol. $43,238-252$.

Fort, A., Delpuech, C., Pernier, J., and Giard, M.-H. (2002). Early auditoryvisual interactions in human cortex during nonredundant target identification. Brain Res. Cogn. Brain Res. 14, 20-30.

Fort, A., and Giard, M. (2004). "Multiple electrophysiological mechanisms of audiovisual integration in human perception," in The Handbook of Multisensory Processes, eds G. A. Calvert, C. Spence, and B. E. Stein (Cambridge, MA: MIT Press), 503-515.

Foxe, J. J., and Molholm, S. (2009). Ten years at the multisensory forum: musings on the evolution of a field. Brain Topogr. 21, 149-154.

Fujisaki, W., Shimojo, S., Kashino, M., and Nishida, S. (2004). Recalibration of audiovisual simultaneity. Nat. Neurosci. 7, 773-778.

Hay-McCutcheon, M. J., Pisoni, D. B., and Hunt, K. K. (2009). Audiovisual

to develop a unity assumption (Welch and Warren, 1980) and to anticipate temporal synchrony relations between vision and audition.

To conclude, we confirmed infants' sensitivity to temporal synchrony relations in ecologically valid, non-speech stimuli, and demonstrated temporal interactions between vision and audition in the infant brain toward perceptual fusion although the magnitude of the asynchrony did not allow for simultaneity perception. The present results suggest anticipatory mechanisms and predictive capacities as to audiovisual temporal synchrony relations in infants as young as 6 months of age.

\section{ACKNOWLEDGMENTS}

We are grateful to all members of the BabyLab at the Max Planck Institute for Human Development, Berlin, and to all infants and parents who participated in this study. We wish to thank Ulman Lindenberger for supporting this research and for his helpful comments and Julia Delius for language editing.

asynchrony detection and speech perception in hearing-impaired listeners with cochlear implants: a preliminary analysis. Int. J. Audiol. 48, 321-333.

Hill Karrer, J., Karrer, R., Bloom, D., Chaney, L., and Davis, R. (1998). Event-related brain potentials during an extended visual recognition memory task depict delayed development of cerebral inhibitory processes among 6month-old infants with Down syndrome. Int. J. Psychophysiol. 29, 167-200.

Hyde, D. C., Jones, B. L., Flom, R. and Porter, C. L. (2011). Neural signatures of face-voice synchrony in 5-month-old human infants. Dev. Psychobiol. 53, 359-370.

Hyde, D. C., Jones, B. L., Porter, C. L. and Flom, R. (2010). Visual stimulation enhances auditory processing in 3-month-old infants and adults. Dev. Psychobiol. 52, 181-189.

Junghöfer, M., Elbert, T., Tucker, D. M., and Braun, C. (1999). The polar average reference effect: a bias in estimating the head surface integral in EEG recording. Clin. Neurophysiol. 110, 1149-1155.

Karrer, R., and Monti, L. A. (1995). Event-related potentials of 4-6week-old infants in a visual recognition memory task. Electroencephalogr. Clin. Neurophysiol. 94, 414-424.

Kopp, F., and Lindenberger, U. (2011). Effects of joint attention on longterm memory in 9-month-old infants: an event-related potentials study. Dev. Sci. 14, 660-672.

Kopp, F., and Lindenberger, U. (2012). Social cues at encoding affect memory in four-month-old infants. Soc. Neurosci. 7, 458-472.
Lewkowicz, D. J. (1986). Developmental changes in infants' bisensory response to synchronous durations. Infant Behav. Dev. 9, 335-353.

Lewkowicz, D. J. (1992). Infants' response to temporally based intersensory equivalence: the effect of synchronous sounds on visual preferences for moving stimuli. Infant Behav. Dev. 15, 297-324.

Lewkowicz, D. J. (1996). Perception of auditory-visual temporal synchrony in human infants. J. Exp. Psychol. Hum. Percept. Perform. 22, 1094-1106.

Lewkowicz, D. J. (2000). The development of intersensory temporal perception: an epigenetic systems/limitations view. Psychol. Bull. 126, 281-308.

Lewkowicz, D. J. (2010). Infant perception of audiovisual speech synchrony. Dev. Psychol. 46, 66-77.

Lewkowicz, D. J., Leo, I., and Simion, F. (2010). Intersensory perception at birth: newborns match nonhuman primate faces and voices. Infancy 15 , 46-60.

Lewkowicz, D. J., Sowinski, R., and Place, S. (2008). The decline of crossspecies intersensory perception in human infants: underlying mechanisms and its developmental persistence. Brain Res. 1242, 291-302.

Macaluso, E., George, N., Dolan, R., Spence, C., and Driver, J. (2004). Spatial and temporal factors during processing of audiovisual speech: a PET study. Neuroimage 21, 725-732.

Miller, L. M., and D'Esposito, M. D. (2005). Perceptual fusion and stimulus coincidence in the cross-modal integration of speech. J. Neurosci. 25, 5884-5893. 
Navarra, J., Hartcher-O’Brien, J., Piazza, E., and Spence, C. (2009). Adaptation to audiovisual asynchrony modulates the speeded detection of sound. Proc. Natl. Acad. Sci. U.S.A. 106, 9169-9173.

Navarra, J., Vatakis, A., Zampini, M., Soto-Faraco, S., Humphreys, W., and Spence, C. (2005). Exposure to asynchronous audiovisual speech extends the temporal window for audiovisual integration. Brain Res. Cogn. Brain Res. 25, 499-507.

Petrini, K., Dahl, S., Rocchesso, D., Waadeland, C. H., Avanzini, F., Puce, A., et al. (2009a). Multisensory integration of drumming actions: musical expertise affects perceived audiovisual synchrony. Exp. Brain Res. 198, 339-352.

Petrini, K., Russell, M., and Pollick, F. (2009b). When knowing can replace seeing in audiovisual integration of actions. Cognition 110, 432-439.

Pilling, M. (2009). Auditory eventrelated potentials (ERPs) in audiovisual speech perception. J. Speech Lang. Hear. Res. 52, 1073-1081.

Putzar, L., Goerendt, I., Lange, K., Rösler, F., and Röder, B. (2007). Early visual deprivation impairs multisensory interactions in humans. Nat. Neurosci. 10, 1243-1245.

Snyder, K., Webb, S. J., and Nelson, C. A. (2002). Theoretical and methodological implications of variability in infant brain response during a recognition memory paradigm. Infant Behav. Dev. 25, 466-494.

Spelke, E. S. (1979). Perceiving bimodally specified events in infancy. Dev. Psychol. 15, 626-636.

Spence, C., and Squire, S. (2003). Multisensory integration: maintaining the perception of synchrony. Curr. Biol. 13, R519-R521.

Stekelenburg, J. J., and Vroomen, J. (2007). Neural correlates of multisensory integration of ecologically valid audiovisual events. J. Cogn. Neurosci. 19, 1964-1973.

Stets, M., Stahl, D., and Reid, V. M. (2012). A meta-analysis investigating factors underlying attrition rates in infant ERP studies. Dev. Neuropsychol. 37, 226-252.

Stevenson, R. A., VanDerKlok, R. M., Pisoni, D. B., and James, T. W. (2011). Discrete neural substrates underlie complementary audiovisual speech integration processes. Neuroimage 55, 1339-1345.

Talsma, D., Senkowski, D., Soto-Faraco, S., and Woldorff, M. G. (2010). The multifaceted interplay between attention and multisensory integration. Trends Cogn. Sci. (Regul. Ed.) 14, 400-410.

Talsma, D., Senkowski, D., and Woldorff, M. G. (2009). Intermodal attention affects the processing of the temporal alignment of audiovisual stimuli. Exp. Brain Res. 198, 313-328.
Thomas, D. G., Whitaker, E., Crow C. D., Little, V., Love, L., Lykins, M. S., et al. (1997). Event-related potential variability as a measure of information storage in infant development. Dev. Neuropsychol. 13, 205-232.

van Wassenhove, V., Grant, K. W., and Poeppel, D. (2005). Visual speech speeds up the neural processing of auditory speech. Proc. Natl. Acad. Sci. U.S.A. 102, 1181-1186.

Vatakis, A., Navarra, J., Soto-Faraco, S. and Spence, C. (2007). Temporal recalibration during asynchronous audiovisual speech perception. Exp. Brain Res. 181, 173-181.

Vatakis, A., and Spence, C. (2006). Audiovisual synchrony perception for music, speech, and object actions. Brain Res. 1111, 134-142.

Vroomen, J., and Stekelenburg, J. J. (2009). Visual anticipatory information modulates multisensory interactions of artificial audiovisual stimuli. J. Cogn. Neurosci. 22, 1583-1596.

Wallace, M. T., Perrault, T. J. Jr., Hairston, W. D., and Stein, B. E. (2004). Visual experience is necessary for the development of multisensory integration. J. Neurosci. 24, 9580-9584.

Webb, S. J., Long, J. D., and Nelson, C. A. (2005). A longitudinal investigation of visual event-related potentials in the first year of life. Dev. Sci. 8, 605-616.
Welch, R. B., and Warren, D. H. (1980). Immediate perceptual response to intersensory discrepancy. Psychol. Bull. 88, 638-667.

Wunderlich, J. L., Cone-Wesson, B. K., and Shepherd, R. (2006). Maturation of the cortical auditory evoked potentials in infants and young children. Hear. Res. 212, 185-202.

Conflict of Interest Statement: The authors declare that the research was conducted in the absence of any commercial or financial relationships that could be construed as a potential conflict of interest.

Received: 12 September 2012; accepted: 03 January 2013; published online: 21 January 2013.

Citation: Kopp F and Dietrich C (2013)

Neural dynamics of audiovisual synchrony and asynchrony perception in 6month-old infants. Front. Psychology 4:2. doi: 10.3389/fpsyg.2013.00002

This article was submitted to Frontiers in Developmental Psychology, a specialty of Frontiers in Psychology.

Copyright (C) 2013 Kopp and Dietrich. This is an open-access article distributed under the terms of the Creative Commons Attribution License, which permits use, distribution and reproduction in other forums, provided the original authors and source are credited and subject to any copyright notices concerning any third-party graphics etc. 